\title{
Elastic Softness of Hybrid Lead Halide Perovskites
}

\author{
A. C. Ferreira, ${ }^{1,2}$ A. Létoublon, ${ }^{2}$ S. Paofai, ${ }^{3}$ S. Raymond, ${ }^{4}$ C. Ecolivet, ${ }^{5}$ B. Rufflé, ${ }^{6}$ S. Cordier, ${ }^{3}$ C. Katan, ${ }^{3}$ \\ M. I. Saidaminov, ${ }^{7, *}$ A. A. Zhumekenov, ${ }^{7}$ O. M. Bakr, ${ }^{7}$ J. Even, ${ }^{2}$ and P. Bourges ${ }^{1}$ \\ ${ }^{1}$ Laboratoire Léon Brillouin, CEA-CNRS, Université Paris-Saclay, CEA Saclay, 91191 Gif-sur-Yvette, France \\ ${ }^{2}$ Univ Rennes, INSA Rennes, CNRS, Institut FOTON - UMR 6082, F-35000, Rennes, France \\ ${ }^{3}$ Univ Rennes, ENSCR, INSA Rennes, CNRS, ISCR (Institut des Sciences Chimiques de Rennes) - UMR 6226, \\ F-35000, Rennes, France \\ ${ }^{4}$ Univ. Grenoble Alpes, CEA, INAC, MEM, 38000 Grenoble, France \\ ${ }^{5}$ Univ Rennes, CNRS, IPR (Institut de Physique de Rennes) - UMR 6251, F-35000 Rennes, France \\ ${ }^{6}$ Laboratoire Charles Coulomb (L2C), UMR 5221 CNRS-Université de Montpellier, Montpellier, FR-34095, France \\ ${ }^{7}$ King Abdullah University of Science and Technology (KAUST), KAUST Catalysis Center, KAUST Solar Center, \\ Physical Sciences and Engineering Division (PSE), Thuwal 23955-6900, Saudi Arabia
}

(Received 26 December 2017; published 22 August 2018)

\begin{abstract}
Much recent attention has been devoted towards unraveling the microscopic optoelectronic properties of hybrid organic-inorganic perovskites. Here we investigate by coherent inelastic neutron scattering spectroscopy and Brillouin light scattering, low frequency acoustic phonons in four different hybrid perovskite single crystals: $\mathrm{MAPbBr}_{3}, \mathrm{FAPbBr}_{3}, \mathrm{MAPbI}_{3}$, and $\alpha$-FAPbI $\mathrm{F}_{3}$ (MA: methylammonium, FA: formamidinium). We report a complete set of elastic constants characterized by a very soft shear modulus $C_{44}$. Further, a tendency towards an incipient ferroelastic transition is observed in $\mathrm{FAPbBr}_{3}$. $\mathrm{We}_{\text {observe a }}$ systematic lower sound group velocity in the technologically important iodide-based compounds compared to the bromide-based ones. The findings suggest that low thermal conductivity and hot phonon bottleneck phenomena are expected to be enhanced by low elastic stiffness, particularly in the case of the ultrasoft $\alpha-\mathrm{FAPbI}_{3}$.
\end{abstract}

DOI: 10.1103/PhysRevLett.121.085502

Motivated by environmental and energy issues, hybrid organolead perovskites (HOP) have drawn a lot of interest in the field of photovoltaic cells [1-7]. Currently, the state of the art of HOP solar cells is based on alloys where methylammonium (MA) and formamidinium (FA) are both present in the same structure and $c a .10 \%$ are replaced by rubidium $(\mathrm{Rb})$ and caesium $(\mathrm{Cs})$ atoms, together with concomitant alloying of iodide (I)/bromide (Br) halogens [8], now reaching power conversion efficiencies (PCEs) in excess of 22\% [9]. The rapid emergence and success of hybrid perovskites is widely attributed to various features such as low cost and low temperature processing, suitable optical band gap (especially for iodide based HOPs), superb optical absorption across the visible spectrum, low exciton binding energies, and long charge-carrier diffusion lengths. Much recent attention has been devoted towards HOPs charge-carrier features [10-13] and previous experimental studies have successfully evidenced the influence of crystal structure on their optoelectronic properties, as exemplified with the effect of octahedral tilt on the band gap $[14,15]$. However, the origin of the softness of HOPs, compared to classic semiconductors, and its influence on their charge-carrier dynamics is still lacking a comprehensive understanding and systematic experimental studies. Furthermore, in perovskite based thin film solar cells, device performance is deeply affected by film quality and fabrication processing (morphological effects, grain boundaries, etc.), which makes it difficult to study the intrinsic properties of HOPs. Single crystals, on the other hand, provide the ideal platform to uncover their fundamental limits. In this work, we have investigated low frequency structural excitations in the cubic phases of the most relevant compounds implemented in HOPs, namely, $\mathrm{MAPbBr}_{3}, \mathrm{FAPbBr}_{3}, \mathrm{MAPbI}_{3}$, and $\alpha-\mathrm{FAPbI}_{3}$, in their single crystal form. For additional information regarding structure and crystal growth please refer to the Supplemental Material [16]. We report a complete set of elastic constants, via the corresponding sound velocities, and we relate the results to the lower thermal conductivity found in HOP compounds and the hot phonon bottleneck hypothesis proposed for such systems. For that purpose, dispersions of the acoustic phonons have been measured around main Bragg reflections using inelastic neutron scattering (INS). Complementary Brillouin light scattering (BLS) experiments have also been used to determine sound velocities in the bromide-based compounds. The experimental conditions and procedure of both techniques are detailed in the Supplemental Material [16].

Figure 1 shows the low energy INS spectra of transverse acoustic (TA) phonons in $\mathrm{MAPbBr}_{3}, \mathrm{FAPbBr}_{3}, \mathrm{MAPbI}_{3}$, 

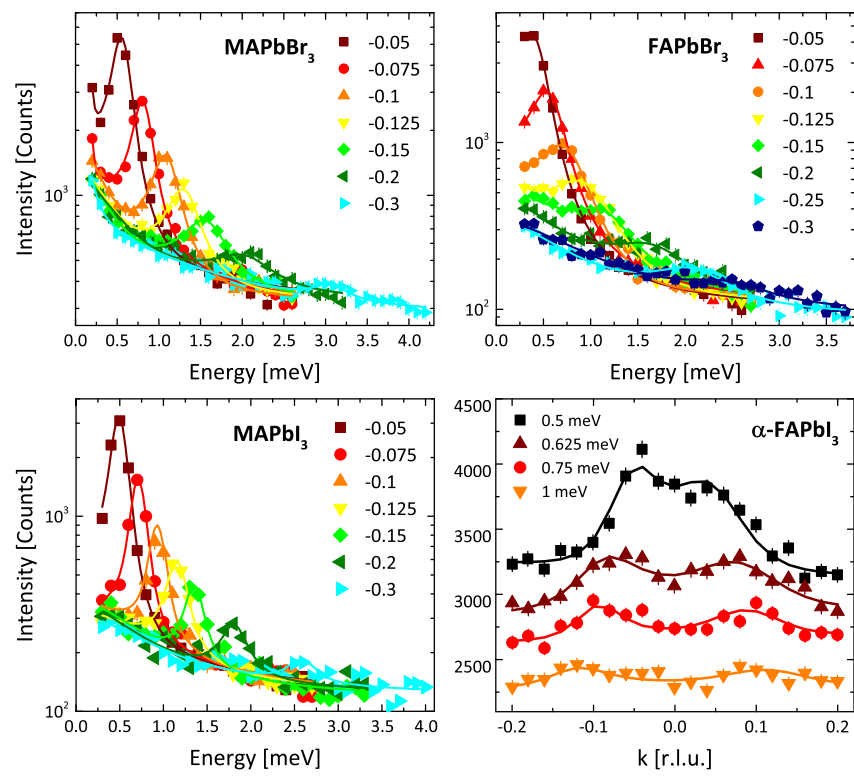

FIG. 1. Transverse acoustic (TA) phonon spectra measured by inelastic neutron scattering in the cubic phase of (a) $\mathrm{MAPbBr}_{3}$, (b) $\mathrm{FAPbBr}_{3}$, and (c) $\mathrm{MAPbI}_{3}$ for different $Q$ positions going away from the $(002) \equiv(200)$ Bragg peak [i.e., $\mathrm{Q}=(k k 2)$ ]; and of (d) $\alpha$-FAPbI ${ }_{3}$ for different energy values. With the exception of $\mathrm{MAPbI}_{3}(340 \mathrm{~K})$, all other compounds were studied at room temperature (RT).

and $\alpha-\mathrm{FAPbI}_{3}$, measured around the Bragg reflection (200). Additional data around other Bragg reflections, (110) and (111), can be found in the Supplemental Material [16]. We performed both constant energy and $Q$ scans at the main Bragg positions. Using the (200) position as an example, longitudinal (LA) and transverse (TA) acoustic modes were measured at different reciprocal space positions $Q=(200)+q$ in HKL units, with $q$ along (LA) and perpendicular (TA) to [200] (for TA $q$ is parallel to [011]).
Clear acoustic phonon modes are observed on top of a background. As shown by constant $Q$ scans, the background results from large quasielastic signals, likely coming from incoherent scattering of hydrogen atom excitations present in the MA/FA molecules, as it has been previously studied on powder samples in $\mathrm{MAPbBr}_{3}$ [20]. Energy scans performed at various momentum vectors around the Bragg positions revealed that the quasielastic signal occurring in the energy window of the acoustic phonons is almost independent of the sample orientation and weakly dependent of the longitudinal momentum. In constant-energy scans [e.g., Fig. 1(d)], the incoherent quasielastic signal corresponds to a flat contribution, whereas acoustic phonons show up as a double peak structure at symmetric positions of the Bragg reflection. As a result, even though the background is relatively large, one can easily separate it from the dispersing and symmetric phonon modes. All neutron spectra have been fitted as described in the Supplemental Material [16], from which the phonon energy can be extracted.

By varying the distance $q$ to the nearest Bragg peak, $\Gamma$ point, one can draw the dispersion curves, which are reported in Figs. 2(a)-2(b) and in Supplemental Material [16]. Each subfigure represents measurements at a different Bragg position or propagation direction. We derive the phonon sound velocity $v$ from linear regressions along most directions and then, with simple rules of elasticity in cubic systems [21], readily determine the corresponding elastic constants for the four perovskite compounds. Figures 2(a) and 2(b) specifically show the sound velocities of transverse and longitudinal acoustic phonons around the (002) Bragg reflection. These respectively yield $C_{44}$ and $C_{11}$ in a rather direct way $\left(C=\rho v^{2}\right)$ which are then plotted as a function of the lattice constant in Fig. 2(c). A summary of the determined sound velocities by INS for all four HOP compounds is given in the Supplemental Material [16] and the extracted elastic constants are in Table I. Considering
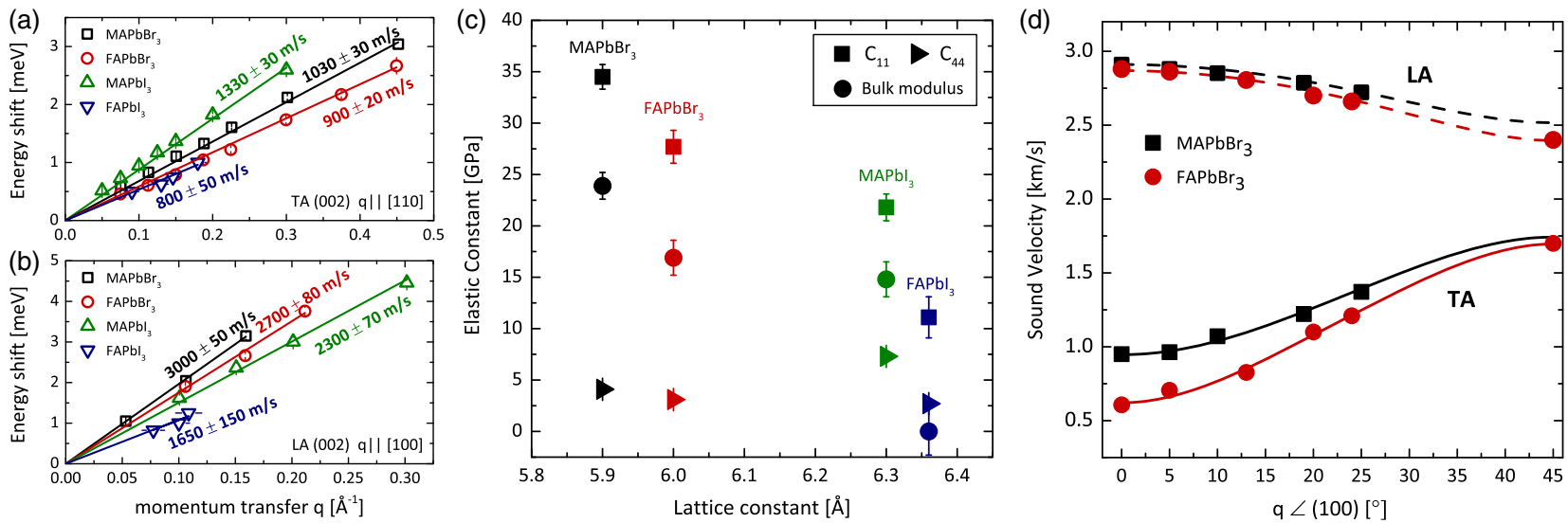

FIG. 2. Acoustic phonon dispersion curves of the four HOP (a) TA and (b) LA phonons at around the (002) Bragg reflection, measured by INS. (c) Elastic constants $C_{11}$ and $C_{44}$ as well as the bulk modulus $K$ behavior as a function of the cubic lattice constant of each compound. (d) Sound velocity diagram for $\mathrm{FAPbBr}_{3}$ and $\mathrm{MAPbBr}_{3}$, as determined by Brillouin light scattering. The velocity is given as a function of the angle between the direction of measurement and the [100] direction. 
TABLE I. Summary of the elastic properties at RT for $\mathrm{MAPbBr}_{3}, \mathrm{FAPbBr}_{3}$, and $\alpha-\mathrm{FAPbI}_{3}$ and at $340 \mathrm{~K}$ for $\mathrm{MAPbI}_{3}$, as measured by inelastic neutron Sscattering (INS) and Brillouin scattering (BS). In $\alpha-\mathrm{FAPbI}_{3}$, the bulk modulus is found: $K=0.1 \pm 2.3 \mathrm{GPa}$ (we here only quote the positive range which is only physically meaningful).

\begin{tabular}{|c|c|c|c|c|c|c|c|c|}
\hline \multirow[b]{2}{*}{ Elastic constant } & \multicolumn{2}{|c|}{$\mathrm{MAPbBr}_{3}$} & \multicolumn{2}{|c|}{$\mathrm{FAPbBr}_{3}$} & \multicolumn{2}{|c|}{$\mathrm{MAPbI}_{3}$} & \multicolumn{2}{|c|}{$\mathrm{FAPbI}_{3}$} \\
\hline & INS & BS & INS & $\mathrm{BS}$ & INS & $\mathrm{BS}$ & INS & $\mathrm{BS}$ \\
\hline$C_{11}[\mathrm{GPa}]$ & $34.5 \pm 1.2$ & $32.2 \pm 0.2$ & $27.7 \pm 1.6$ & $31.2 \pm 0.2$ & $21.8 \pm 1.3$ & $\mathrm{n} / \mathrm{a}$ & $11.1 \pm 2.0$ & $\mathrm{n} / \mathrm{a}$ \\
\hline$C_{44}[\mathrm{GPa}]$ & $4.1 \pm 0.2$ & $3.4 \pm 0.1$ & $3.1 \pm 0.1$ & $1.5 \pm 0.1$ & $7.3 \pm 0.3$ & $\mathrm{n} / \mathrm{a}$ & $2.7 \pm 0.3$ & $\mathrm{n} / \mathrm{a}$ \\
\hline$C_{12}[\mathrm{GPa}]$ & $18.5 \pm 2.0$ & $9.1 \pm 0.8$ & $11.5 \pm 2.4$ & $9.4 \pm 0.5$ & $11.3 \pm 3.1$ & $\mathrm{n} / \mathrm{a}$ & $-5.5 \pm 2.2$ & $\mathrm{n} / \mathrm{a}$ \\
\hline Bulk modulus [GPa] & $23.9 \pm 1.3$ & $16.8 \pm 0.1$ & $16.9 \pm 1.7$ & $16.7 \pm 0.3$ & $14.8 \pm 1.7$ & $\mathrm{n} / \mathrm{a}$ & $0.0 \pm 2.4$ & $\mathrm{n} / \mathrm{a}$ \\
\hline Anisotropy, A & $0.52 \pm 0.005$ & $0.29 \pm 0.01$ & $0.38 \pm 0.03$ & $0.14 \pm 0.01$ & $1.38 \pm 0.22$ & $\mathrm{n} / \mathrm{a}$ & $0.4 \pm 0.2$ & $\mathrm{n} / \mathrm{a}$ \\
\hline$L / T$ ratio & $8.4 \pm 0.5$ & $9.5 \pm 0.3$ & $8.9 \pm 0.7$ & $20.8 \pm 1.4$ & $3.0 \pm 0.2$ & $\mathrm{n} / \mathrm{a}$ & $4.3 \pm 0.9$ & $\mathrm{n} / \mathrm{a}$ \\
\hline
\end{tabular}

the obtained elastic constants one can next calculate the bulk modulus $K=1 / 3\left(C_{11}+2 C_{12}\right)$ [21], Zener anisotropy index $A=2 C_{44} /\left(C_{11}-C_{12}\right) \quad$ [22] and longitudinaltransverse $(L / T)$ ratio for all systems (see Supplemental Material [16]). In $\mathrm{MAPbBr}_{3}$ and $\mathrm{MAPbI}_{3}$, the sound velocities or bulk modulus recently obtained from highresolution inelastic x-ray scattering measurements [23,24], although with less accuracy, agree with our findings.

Immediately we note that $C_{12}$ in $\alpha-\mathrm{FAPbI}_{3}$ is negative, but still it respects the necessary Born elastic stability criteria for cubic systems: $C_{11}-\mathrm{C}_{12}>0$; $\mathrm{C}_{11}+2 \mathrm{C}_{12}>0$ and $\mathrm{C}_{44}>0$ [25,26]. A negative $C_{12}$ simply implies that a cubic material, when uniaxially compressed along a [100] direction, will contract in the other two directions ([010] and [001]) and in that way, try to maintain an isotropic structure. Nonetheless, the negative $C_{12}$ together with the very low bulk modulus, confirms the very unstable nature of $\alpha-\mathrm{FAPbI}_{3}$ (whose metastable single crystals last less than a week in the $\alpha$ phase [27]) and why it has actually been paired with MA, Rb, and Cs for better performing photovoltaic devices. As shown in Table I, the four perovskite compounds exhibit an overall sizeable elastic anisotropic nature $(A \neq 1)$, which can be mostly attributed to the very low shear modulus $C_{44} \cdot \mathrm{MAPbI}_{3}$ stands out with its relatively higher (around double) $C_{44}$ which results in a discrepant, although still anisotropic, Zener index. However, it should be noted that the measurements were performed at $340 \mathrm{~K}$, just above the transition to the cubic phase [28], resulting in an additional anharmonicity effect. The remarkably low shear moduli are much more evident when compared to the ones of classical semiconducting photovoltaic materials such as $\mathrm{Si}, \mathrm{GaAs}$, or $\mathrm{AlAs}$, where the elastic constants are in the $10^{2}$ Gpa order [29]. It is believed that the rotation and tilt of the corner sharing $\mathrm{PbX}_{6}$ octahedra [30] could be responsible for the particularly low shear modulus and large anisotropy in HOPs.

As evidenced in Fig. 2(c), $C_{11}$ and $K$ decrease noticeably with increasing lattice constant. These two quantities are, therefore, lower in iodide-based systems compared with bromide ones, especially in $\alpha-\mathrm{FAPbI}_{3}$ where they are a third of its bromide counterpart. This indicates a structural instability when the lattice parameter exceeds $\sim 6.4 \AA$. The relatively higher bulk modulus in MA-based compounds should be related to a steric effect, where the more symmetric and rotating MA molecules lead to more compact structures, which in turn results in larger binding elastic interactions. It is worth emphasizing that, by construction, the acoustic branches are defining the lowest zone boundary phonons and thus, related to the lowest peaks of the phonon density of states. That also implies that the Debye temperature is very small (i.e., about $30 \mathrm{~K}$ ). Moreover, as a function of lattice constant, this quantity exhibits the same trend as for $C_{11}$ and the bulk modulus. The observation that iodide materials are softer than bromide materials, as well as FA-based compounds versus MA-based ones is consistent with recent static nanoindentation measurements of the Young modulus [31,32], though $\alpha-\mathrm{FAPbI}_{3}$ was not explored there (see table in the Supplemental Material [16] for comparison). In contrast, recent pulse-echo ultrasonic measurements at low frequency $(10 \mathrm{MHz})$ and in the $140-350 \mathrm{~K}$ temperature range [33], show larger sound velocities for $\mathrm{MAPbI}_{3}$ than for $\mathrm{MAPbBr}_{3}$. However, the results of Anusca et al. [33] are also in disagreement with other ultrasonic studies [34,35]. Yet it should be noted that the sound velocity attenuation is very large at $10 \mathrm{MHz}$ and strongly affected by the structural phase transitions.

Brillouin light scattering on the other hand, allows exploring the same properties in the $\mathrm{GHz}$ range, intermediate between ultrasonic and neutron scattering measurements. Sound velocities were measured in both bromide compounds at room temperature (RT) using Brillouin light scattering, with a setup in the [100], [010] base plane [34]. Five different incidence angles between the normal $\left(0^{\circ}\right)$ and the Brewster angle $\left(25^{\circ}\right)$ are reported in Fig. 2(c) with the observation of quasilongitudinal and quasitransverse acoustic modes. A measurement along the cubic diagonal [110] is also shown. For both bromide compounds, a good agreement between INS and BLS longitudinal sound velocities is observed (see Supplemental Material [16]). The same applies for the transverse mode in $\mathrm{MAPbBr}_{3}$ [34], within a $5 \%$ difference. In contrast, a $30 \%$ difference is observed for the same 

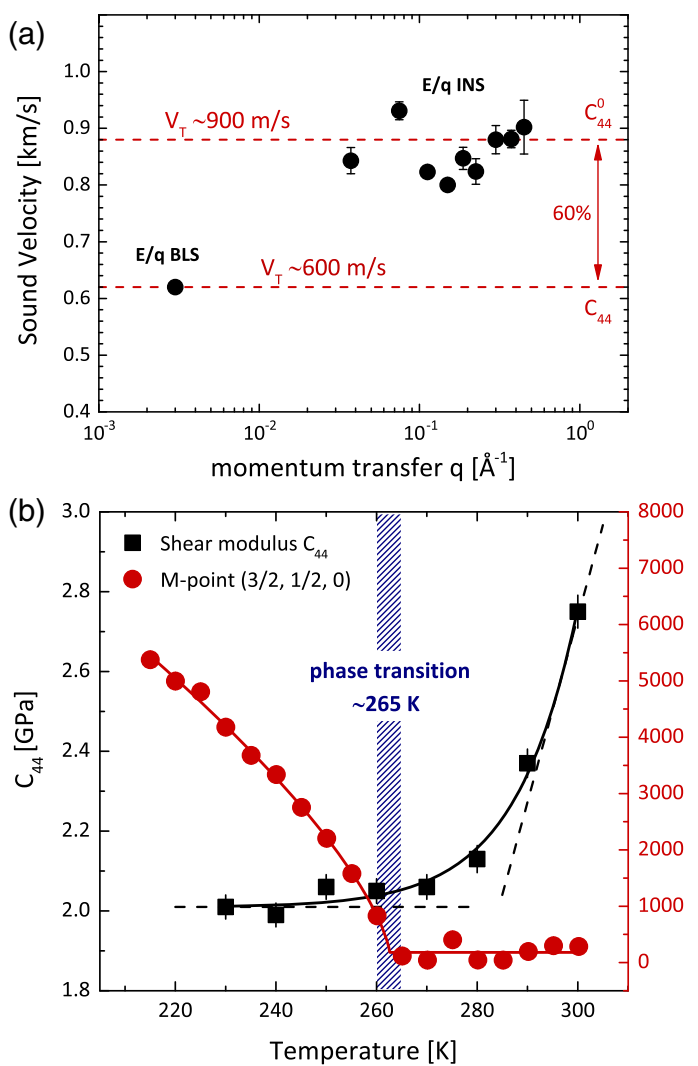

FIG. 3. Softening of $C_{44}$ in $\mathrm{FAPbBr}_{3}$ (a) as function of $q$ (at RT) and (b) as a function of temperature $(230-300 \mathrm{~K})$, around the (002) Bragg reflection. Also in (b) the Bragg $M$ point intensity (red) across a similar a temperature range. The bare elastic constant, $C_{44}^{0}$, represents the elastic properties without the influence of the (incipient) phase transition. Note that the $q$ scale is logarithmic to underline the broad $q$ range covered by both experimental techniques.

mode in $\mathrm{FAPbBr}_{3}$. This is emphasized in Fig. 3(a) where the sound velocity is presented as a function of $q$. One can clearly observe the phonon softening of the transverse mode at lower $q$, in the BLS regime, which indicates a tendency towards a ferroelastic phase transition [36]. Recording that this specific sound velocity is related to the $C_{44}$ elastic constant as $C_{44}=\rho v^{2}$ (where $\rho=4087 \mathrm{~kg} / \mathrm{cm}^{3}$ is the density of $\mathrm{FAPbBr}_{3}$ ), it means that a $30 \%$ softening of the sound velocity corresponds to a $60 \%$ renormalization of $C_{44}$. Interestingly, the values obtained by laser ultrasonics [35], where a set of elastic constants was also given for $\mathrm{MAPbBr}_{3}$, are in good agreement with our BLS results and a similar softening of $\mathrm{C}_{44}$ is observed too.

The softening of the shear modulus $C_{44}$ is typically related to the proximity of a ferroelastic transition [36] which, in this case, can be only incipient. To test that possibility, we have performed a temperature study of the respective acoustic branch in $\mathrm{FAPbBr}_{3}$, using INS at constant $Q=(2,0.025,0.025)$. Indeed, as one can observe in Fig. 3(b), $C_{44}$ is drastically reduced as soon as we start to cool down the sample below RT. However, that effect is blocked once we reach $\sim 260 \mathrm{~K}$. Actually, at around $265 \mathrm{~K}, \mathrm{FAPbBr}_{3}$ additionally undergoes a cubic to tetragonal 2nd order phase transition, characterized by the doubling of the unit cell of the crystal. This is revealed by the appearance of a Bragg reflection at the $M$ point, e.g., $Q=$ $(3 / 2,1 / 2,0)$ [Fig. 3(b)]. It is known that lead perovskites exhibit structural instabilities at both the $M$ and $R$ points [37]. However, such antiferrodistorsive instabilities cannot produce in the disordered phase (here above $265 \mathrm{~K}$ ) any softening of the elastic constants [36]. Therefore, the softening of $C_{44}$ at high temperature is due to an independent tendency towards a ferroelastic instability, which is blocked by the cubic to tetragonal phase transition, limiting ferroelastic pretransitional effects compared to what would be expected for a full phase transition [36]. In Fig. 3(b), one can easily see how the two processes coincide. By linearly extrapolating the softening rate at high temperature (before it is blocked), one can estimate that such a ferroelastic transition would occur around $240 \pm 20 \mathrm{~K}$. Consistently also with an aborted ferroelastic instability, a very modest phonon broadening is observed with decreasing temperature from 300 to $230 \mathrm{~K}$ (see Supplemental Material [16]).

HOPs are characterized by high electron and hole free charge carrier mobility at room temperature. At high temperature ranges such as RT (10 times the Debye temperature), the electronic mobility, $\mu$, is typically governed by phonon scattering, via electron-phonon coupling (Fröhlich phonon emission). The whole phonon spectrum will then contribute to the electronic scattering rate, $1 / \tau(\mu$ is proportional to the electronic relaxation time $\tau$ ). However, for intravalley electron bands of direct gap semiconductors like the 3D HOP, the scattering rate should also be enhanced by collisions with low energy longitudinal acoustic phonons. When the acoustic phonon contribution is considered, the electronic scattering time $\tau$ is expected to be proportionnal to the average squared longitudinal sound velocities [38], i.e., proportional to the average elastic constants such as $C_{11}$ or the bulk modulus $K$ that are shown in Fig. 2(c). The contributions to the carrier mobilities related to interactions with acoustic phonons are thus predicted to be strongly different between iodide- and bromide-based compounds, but such a large difference in carrier mobilities is not experimentally observed [27,39]. Instead, it rather shows that the carrier mobilities are limited by other processes, namely, interactions with optical phonons. Such a conclusion is corroborated by emission line broadening results [40,41], and it confirms that scattering from longitudinal optical phonons could be the dominant source of electron-phonon coupling near RT.

It is necessary, however, to consider the acoustic phonons in order to explain other optoelectronic properties that Fröhlich optical phonon emission does not account for. More precisely, a significant hot-phonon bottleneck effect in carrier thermalization is observed in lead halide perovskites $[42,43]$. The effect consists of the up-conversion of acoustic phonons into optical modes, which recycles 
thermal (vibrational) energy, reheating charge carriers and prolonging the cooling period of the carrier-optical phonon system. This helps maintaining a hot carrier population, something that is vital to achieve hot carrier photovoltaic devices and break the Shockley-Queisser limit. At the same time, ultrafast transient absorption measurements reveal two stages of the carrier cooling process [43]. The first one is related to the intrinsic Fröhlich phonon emission mentioned above and does not vary significantly among the various different perovskites. On the other hand, in the second cooling stage, hot carrier-phonon dynamics occur, corresponding to the phonon bottleneck effect $[42,43]$. The various HOP materials studied in the present work show, in fact, very different acoustic phonon densities of states that might affect hot phonon energy relaxations. At RT, the carrier-phonon relaxation rate of that second cooling stage is typically 3-4 times slower in $\alpha-\mathrm{FAPbI}_{3}$, compared with the $\mathrm{MAPbBr}_{3}$ system [43]. This is fully consistent with the difference in elastic constants reported in Fig. 2(c).

Another direct consequence of the lattice softness is the ultralow thermal conductivities reported in perovskites [44]. In any electric semiconductive or insulating material, thermal conductivity $\kappa$ is directly affected by the phonon properties. More specifically, the kinetic theory of phonon transport yields $\kappa=\frac{1}{3} C_{v} v^{2} \tau$, where $C_{v}, v$, and $\tau$ are the phonon specific heat, group velocity, and the scattering rate $[38,44]$. Therefore, since both $\kappa$ and $\tau$ are related to the phonon sound group velocity, it means $\kappa$ is also proportional to the elastic moduli of HOPs [Fig. 2(c)]. One can then associate lower thermal conductivity to lower elastic stiffness $[32,45,46]$. This correlation and the similar behavior of $C_{11}$ and relaxation rates seen above, suggest lower elastic stiffness to be, therefore, an important factor to enhance the hot carrier effect in hybrid perovskites.

In conclusion, our quantitative study of low energy acoustic phonons provides a complete set of elastic constants of various technologically relevant hybrid perovskites, in their cubic phases. We highlight the overall very low shear modulus $C_{44}$ and the significant variation of elastic bulk modulus among them. This way we give a clear picture of the difference in softness between FA and MA based compounds and how it relates to their structural stability and their ultralow thermal conductivities. Moreover, the data presented here strongly support the hypothesis of the hot phonon bottleneck phenomena reported by other authors to explain hot carriers relaxations. Both processes are expected to be enhanced by low elastic stiffness, especially in the case of the ultrasoft $\alpha-\mathrm{FAPbI}_{3}$.

This project has received funding from the European Union's Horizon 2020 programme, through a FET Open research and innovation action under the Grant agreement No. 687008. M. I. S. acknowledges the support of Banting Postdoctoral Fellowship Program, administered by the Government of Canada.
*Present address: Department of Electrical and Computer Engineering, University of Toronto, Toronto, Ontario M5S 3G4, Canada.

[1] A. Kojima, K. Teshima, Y. Shirai, and T. Miyasaka, J. Am. Chem. Soc. 131, 6050 (2009).

[2] D. B. Mitzi, C. D. Dimitrakopoulos, and L. L. Kosbar, Chem. Mater. 13, 3728 (2001).

[3] J. L. Knutson, J. D. Martin, and D. B. Mitzi, Inorg. Chem. 44, 4699 (2005).

[4] N.-G. Park, Mater. Today 18, 65 (2015).

[5] W. S. Yang, J. H. Noh, N. J. Jeon, Y. C. Kim, S. Ryu, J. Seo, and S. I. Seok, Science 348, 1234 (2015).

[6] E. H. Anaraki, A. Kermanpur, L. Steier, K. Domanski, T. Matsui, W. Tress, M. Saliba, A. Abate, M. Grätzel, A. Hagfeldt et al., Energy Environ. Sci. 9, 3128 (2016).

[7] NREL chart, https://www.nrel.gov/pv/assets/images/ efficiency-chart-20180716.jpg, accessed: 16.07.2018.

[8] M. Saliba, T. Matsui, K. Domanski, J.-Y. Seo, A. Ummadisingu, S. M. Zakeeruddin, J.-P. Correa-Baena, W. R. Tress, A. Abate, A. Hagfeldt et al., Science 354, 206 (2016).

[9] W. S. Yang, B.-W. Park, E. H. Jung, N. J. Jeon, Y. C. Kim, D. U. Lee, S. S. Shin, J. Seo, E. K. Kim, J. H. Noh et al., Science 356, 1376 (2017).

[10] Y. Yamada, T. Nakamura, M. Endo, A. Wakamiya, and Y. Kanemitsu, J. Am. Chem. Soc. 136, 11610 (2014).

[11] V. D'Innocenzo, G. Grancini, M. J. Alcocer, A. R. S. Kandada, S. D. Stranks, M. M. Lee, G. Lanzani, H. J. Snaith, and A. Petrozza, Nat. Commun. 5, 3586 (2014).

[12] C. Wehrenfennig, G. E. Eperon, M. B. Johnston, H. J. Snaith, and L. M. Herz, Adv. Mater. 26, 1584 (2013).

[13] L. M. Herz, Annu. Rev. Phys. Chem. 67, 65 (2016).

[14] J. Even, L. Pedesseau, J.-M. Jancu, and C. Katan, J. Phys. Chem. Lett. 4, 2999 (2013).

[15] J. Even, L. Pedesseau, and C. Katan, J. Phys. Chem. C 118, 11566 (2014).

[16] See Supplemental Material at http://link.aps.org/ supplemental/10.1103/PhysRevLett.121.085502 for supporting information about sample preparation, experimental conditions in Brillouin scattering, and additional results and analysis of inelastic neutron scattering experiments which includes Refs. [17-19].

[17] K. Schmalzl, W. Schmidt, S. Raymond, H. Feilbach, C. Mounier, B. Vettard, and T. Bruckel, Nucl. Instrum. Methods Phys. Res., Sect. A 819, 89 (2016).

[18] P. Bourges, M. H. Lemée-Cailleau, P. Launois, C. Ecolivet, H. Cailleau, F. Moussa, and A. Mierzejewski, Phys. Rev. B 54, 15002 (1996).

[19] R. Thurston and K. Brugger, Phys. Rev. 133, A1604 (1964).

[20] I. P. Swainson, C. Stock, S. F. Parker, L. Van Eijck, M. Russina, and J. W. Taylor, Phys. Rev. B 92, 100303(R) (2015).

[21] D. Halliday, R. Resnick, and J. Walker, Fundamentals of Physics, 5th Extended (Wiley, New York, 1997).

[22] C. Zener, Elasticity and Anelasticity of Metals (University of Chicago, Chicago, IL, 1948).

[23] R. Comin, M. K. Crawford, A. H. Said, N. Herron, W. E. Guise, X. Wang, P. S. Whitfield, A. Jain, X. Gong, A. J. McGaughey et al., Phys. Rev. B 94, 094301 (2016). 
[24] A. N. Beecher, O. E. Semonin, J. M. Skelton, J. M. Frost, M. W. Terban, H. Zhai, A. Alatas, J. S. Owen, A. Walsh, and S. J. Billinge, ACS Energy Lett. 1, 880 (2016).

[25] M. Born, in Mathematical Proceedings of the Cambridge Philosophical Society (Cambridge Univ Press, 1940), Vol. 36, pp. 160-172.

[26] F. Mouhat and F.-X. Coudert, Phys. Rev. B 90, 224104 (2014).

[27] A. A. Zhumekenov, M. I. Saidaminov, M. A. Haque, E. Alarousu, S. P. Sarmah, B. Murali, I. Dursun, X.-H. Miao, A. L. Abdelhady, T. Wu et al., ACS Energy Lett. 1, 32 (2016).

[28] P. S. Whitfield, N. Herron, W. E. Guise, K. Page, Y. Q. Cheng, I. Milas, and M. K. Crawford, Sci. Rep. 6, 35685 (2016).

[29] I. Vurgaftman, J. R. Meyer, and L. R. Ram-Mohan, J. Appl. Phys. 89, 5815 (2001).

[30] I. P. Swainson, M. G. Tucker, D. J. Wilson, B. Winkler, and V. Milman, Chem. Mater. 19, 2401 (2007).

[31] Y. Rakita, S. R. Cohen, N. K. Kedem, G. Hodes, and D. Cahen, MRS Commun. 5, 623 (2015).

[32] G. A. Elbaz, W.-L. Ong, E. A. Doud, P. Kim, D. W. Paley, X. Roy, and J. A. Malen, Nano Lett. 17, 5734 (2017).

[33] I. Anusca, S. Balčiūnas, P. Gemeiner, Š. Svirskas, M. Sanlialp, G. Lackner, C. Fettkenhauer, J. Belovickis, V. Samulionis, M. Ivanov et al., Adv. Energy Mater. 7, 1700600 (2017).

[34] A. Létoublon, S. Paofai, B. Rufflé, P. Bourges, B. Hehlen, T. Michel, C. Ecolivet, O. Durand, S. Cordier, C. Katan et al., J. Phys. Chem. Lett. 7, 3776 (2016).
[35] A. M. Lomonosov, X. Yan, C. Sheng, V. E. Gusev, C. Ni, and Z. Shen, Physica Status Solidi (RRL) 10, 606 (2016).

[36] H. Cummins, Phil. Trans. R. Soc. A 293, 393 (1979).

[37] Y. Fujii, S. Hoshino, Y. Yamada, and G. Shirane, Phys. Rev. B 9, 4549 (1974).

[38] R. G. Chambers, Electron in Metals and Semiconductors (Chapman and Hall, London, 1990), p. 112.

[39] M. I. Saidaminov, A. L. Abdelhady, B. Murali, E. Alarousu, V. M. Burlakov, W. Peng, I. Dursun, L. Wang, Y. He, G. Maculan, A. Goriely, T. Wu, O. F. Mohammed, and O. M. Bakr, Nat. Commun. 6, 7586 (2015).

[40] A. D. Wright, C. Verdi, R. L. Milot, G. E. Eperon, M. A. Pérez-Osorio, H. J. Snaith, F. Giustino, M. B. Johnston, and L. M. Herz, Nat. Commun. 7, 11755 (2016).

[41] H. Diab, G. Trippé-Allard, F. Lédée, K. Jemli, C. Vilar, G. Bouchez, V. L. Jacques, A. Tejeda, J. Even, J.-S. Lauret, E. Deleporte, and D. Garrot, J. Phys. Chem. Lett. 7, 5093 (2016).

[42] Y. Yang, D. P. Ostrowski, R. M. France, K. Zhu, J. Van De Lagemaat, J. M. Luther, and M. C. Beard, Nat. Photonics 10, 53 (2016).

[43] J. Yang, X. Wen, H. Xia, R. Sheng, Q. Ma, J. Kim, P. Tapping, T. Harada, T. W. Kee, F. Huang et al., Nat. Commun. 8, 14120 (2017).

[44] M. Wang and S. Lin, Adv. Funct. Mater. 26, 5297 (2016).

[45] A. Pisoni, J. Jaćimović, O. S. Barišić, M. Spina, R. Gaál, L. Forró, and E. Horváth, J. Phys. Chem. Lett. 5, 2488 (2014).

[46] A. Kovalsky, L. Wang, G. T. Marek, C. Burda, and J. S. Dyck, J. Phys. Chem. C 121, 3228 (2017). 